\title{
Diese Suppe sollten wir nicht essen
}

\section{René Mégroz}

Dr. med., Facharzt für Allgemeine Innere Medizin, Mitglied FMH

Im Folgenden stelle ich unter anderem die These auf, dass diese Datenerhebung praktisch ohne Nutzen ist, weil die verlangten Daten längst vorhanden sind. Sie stellt einen erheblichen Ressourcenverschleiss dar. «Früher oder später müssen wir sowieso mitmachen», ist ein oft gehörtes "Argument» für die Teilnahme. Ich möchte ein paar Gegenargumente liefern.

Mit dem Projekt MARS beabsichtigt das Bundesamt für Statistik (BFS) «statistische Datengrundlagen zur Gesundheitsversorgung zur Verfügung zu stellen». Dabei sollen als Ziel verschiedene Fragen geklärt werden. Ausserdem möchte man die Daten für aufsichtsrechtliche Belange nutzen [1]. Laut Versprechen soll das Projekt sogar im Interesse der Ärzte [2] sein. Die Ärztinnen sind zur Lieferung der Daten verpflichtet.

Diese Argumente sprechen gegen die Teilnahme:

1. Die Daten sind schon seit Jahren vorhanden.

2. Der Ressourcenverschleiss ist erheblich: Wir investieren in die Datenlieferung 15,5 Arztjahre.

3. Die Ausgestaltung der aufsichtsrechtlichen Zwecke ist offen.

4. Je länger, desto vorsichtiger sei mit Daten umgegangen.

5. Wir sollten der Aufblähung des Beamtenapparates keinen Vorschub leisten.

\section{Zu 1.}

Die Daten sind schon seit Jahren greifbar bei Steuerämtern, AHV, santésuisse sowie der FMH, überdies existieren noch die RoKo-Zahlen und auch Daten bei den Trustcenter. Eine erneute Lieferung erübrigt sich vollständig für die angeführten Fragestellungen [1].

Jeder Arzt hat eine Steuererklärung inklusive Buchhaltungsdaten abzuliefern. Diese Daten können also verwertet werden (allenfalls müssen dafür die Gesetzesgrundlagen noch geschaffen werden). Gleiches gilt für die AHV. Bei der jeweiligen Ausgleichskasse sind alle Angestellten detailliert erfasst. Wozu nochmals ein Aufwand? Gleichsam können Daten der santésuisse oder der Trustcenter benutzt werden. Hier liegen präzise Daten zur ärztlichen Tätigkeit vor. Die FMH erstellt alljährlich eine umfassende Statistik der Ärzteschaft. Alle Daten liegen möglicherweise in elektronischer Form bereit und harren sozusagen einer Auswertung.
Zu 2.

Der Ressourcenverschleiss ist erheblich. Bei etwa 2300 Jahresstunden [3] einer Ärztin resultiert bei rund 18000 [4] ambulant tätigen Ärzten und gemäss Pilotversuch [5] zwei Stunden Zeitaufwand für den Fragebogen eine Gesamtzeit von 15,5 Arztjahren. Eine Normalarbeitszeit von 42 Wochenstunden angenommen, wären es rund 18 Arztjahre. Der Preis dafür ist beim TARMED-Ansatz 6,9 Mio. Franken, bei einem kostenwahren Ansatz [3] kommen wir auf einen Gegenwert von etwa 11 Mio. Franken.

Für den Einsatz eines solch erheblichen Aufwandes bedarf es besonderer Gründe. Wie gezeigt, sind alle Daten schon vorliegend. Somit ist der grosse Teil der Erhebung eine Doppelspurigkeit - entgegen der Beteuerung des Bundesamtes. Gerade in einer Zeit des Ärztemangels, der Überlastung der Ärzte, der zunehmenden Bürokratie kann kein Verständnis für solch unsinnige Tätigkeiten aufkommen. Wir Ärzte sollten unsere Zeit für die Patienten einsetzen und für unsere Erholung bei ausserordentlich hoher Jahresstundenzahl.

Die an uns gerichtete Aufforderung, längst bekanntes Zahlenmaterial per Dekret nochmals zu erfassen, kommt einem Affront gleich. Auf diese Weise wird mit missliebigen Bittstellern umgegangen: Man lässt sie immer und immer wieder beweisen, was ihr Anliegen ist, lehnt es aber letztlich ab. Hier wird der vermeintliche Nutzen für uns selbst schmackhaft gemacht. Man macht uns glauben, mit dem Zahlenmaterial könnten wir unser Einkommensproblem belegen. Jedoch: Dass die Einkommen der Grundversorger im Lohngefüge aller anderen Ärzte (Ärztinnen im Angestelltenverhältnis, Spezialisten) nicht mehr angemessen sind, ist längst belegt und bedarf keiner weiteren Aufklärung [6].

Überdies ist zu bedenken: Die geforderten Zahlen werden wohl jedes Jahr neu zu erheben sein. Die Frage der Projektleitung [1] legt dies nahe: «Wie sieht die Land- 
schaft der Leistungserbringer in der Gesundheitsversorgung aus und wie entwickelt sie sich?»

Dieser leichtfertige Umgang mit Ressourcen ohne erkennbaren Nutzen ist abzulehnen.

\section{Zu 3.}

Gemäss Art. 59a KVG sind «die Leistungserbringer verpflichtet, den zuständigen Bundesbehörden Daten zur Überwachung von Wirtschaftlichkeit und Qualität der Leistungen bekannt zu geben" [7]. Die Überwachung der WZW-Kriterien erfolgt bisher durch die santésuisse/tarifsuisse. Nun wird auch das BFS noch Auswertungen vornehmen. Diese doppelte Untersuchung hinsichtlich der Einhaltung von WZW-Kriterien ist kaum zu rechtfertigen. Wer bezahlt das und wo ist der Nutzen? Man macht uns ferner weis, die Datenerhebung sei in unserem Interesse. Aber es wird nicht ausgeführt, wie diese Auswertung erfolgt, welche statistischen Modelle zur Anwendung kommen, wer letztlich Sanktionen auferlegt. Eine solche unsorgfältige Anlage müssen wir ablehnen und zum jetzigen Zeitpunkt keinesfalls mitwirken.

Die Kontrollen der santésuisse/tarifsuisse sind bereits ein Dauerthema: Unzählige Ärzte wurden in fragwürdigen Verfahren ungerechtfertigt behelligt, die statistischen Methoden halten nach wie vor wissenschaftlichen Kriterien nicht stand [8].

Eine Überwachung der Tarifanwendung durch eine zu bestimmende Stelle ergibt Sinn und ist durch das Gesetz vorgesehen. Allerdings sollte eine Bedingung für unsere Teilnahme sein, dass auch andere Bezüger von Prämiengeldern in die Kontrollen eingeschlossen werden, nicht nur die Leistungserbringer. Die statistischen Methoden sind interdisziplinär zu bestimmen und zu evaluieren, die «Richter» werden paritätisch gewählt und erfüllen noch festzulegende Qualifikationen. Erst dann kann allenfalls so einem Gremium und der zweckgebundenen Datenerhebung zugestimmt werden. Bis dahin scheint es angebracht, keine Daten zu liefern.

\section{Zu 4.}

Vorsichtiger Umgang mit Daten. Es ist das Gebot der Stunde, mit Daten sorgsam umzugehen. Die digitalen Sammlungen sind ein weithin unterschätztes Problem. Es sollen keine Daten auf Vorrat erfasst werden. Nur diese Daten, die unmittelbar für eine sinnvolle Fragestellung unumgänglich sind, sollen auch angesammelt werden. Beim aktuellen Vorgang des BFS entsteht der Eindruck, dass alles Erdenkliche mal angehäuft wird.

\section{Zu 5.}

Wie viele Stellen müssen geschaffen werden, um die Daten von 18000 Ärzten zu verwalten? Das wissen wir nicht. Wir sollten nicht Hand bieten zur Aufblähung von Verwaltungsvorgängen, deren Nutzen letztlich minimal ist. Die einmal eingestellten Personen wollen auch in den Folgejahren beschäftigt sein und fordern immer neue Aufgaben. Daher: cavete.

Es gibt also gute und ehrenwerte Gründe, diese Aufforderung zur Datenerhebung zu ignorieren. Es ist nicht bloss eine Trotzhaltung, sondern es werden konstruktive Vorschläge gemacht. Mit den ärztlichen Ressourcen sollte sehr sorgsam umgegangen werden, eine $\mathrm{Ab}$ lehnung ist auch im Interesse der Versorgungsqualität. Man darf davon ausgehen, dass es eines Bürgers Recht ist, unverhältnismässige staatliche Aufforderungen zu verweigern.

\section{Literatur}

1 Huguenin J. Projekt MARS - Statistiken der ambulanten Gesundheitsversorgung. Schweiz Ärztezeitung. 2014;95:22/23:859-61. Unter anderem sollen diese Fragen geklärt werden: Ist die medizinische Versorgung angemessen und ausreichend für die Struktur der Bevölkerung? Wie sieht die Landschaft der Leistungserbringer in der Gesundheitsversorgung aus und wie entwickelt sie sich? Wie steht das Leistungsangebot im Verhältnis zur Versorgungsnachfrage? Wie entwickelt sich die Inanspruchnahme von bestimmten Leistungen? Werden stationäre Behandlungen durch ambulante ersetzt? Was sind die Auswirkungen für das Gesundheitssystem und für die Patientinnen und Patienten? Wie kann beispielsweise der Zugang zur medizinischen Grundversorgung in hoher Qualität gesichert werden? Der Gesetzesauftrag zur Erhebung von Daten der Leistungserbringer zu aufsichtsrechtlichen Zwecken ist in KVG Art. 22a festgehalten. Dadurch sollen die Wirtschaftlichkeit und Qualität der Leistungen überwacht werden.

2 Es sind stets beide Geschlechter gemeint.

3 Mégroz R. Was kostet eine Stunde Arzt? Wirtschaftliche und andere Überlegungen. Schweiz Ärztezeitung. 2004;85(24):1286-90.

418128 Ärzte arbeiten im ambulanten Sektor gemäss: Hostettler S, Kraft E. Zuwanderung grundlegend für Versorgungssystem. Schweiz Ärztezeitung. 2016;97(12-13):448-53.

5 Huguenin J. Auf dem Weg zum MARS, Schweiz Ärztezeitung. 2016;97(7):247.

6 Z.B. «150» in Ars medici 2008;1; seit Jahren werden RoKo-Zahlen geliefert und publiziert, die das belegen.

7 Die Leistungserbringer sind verpflichtet, den zuständigen Bundesbehörden die Daten bekannt zu geben, die benötigt werden, um die Anwendung der Bestimmungen dieses Gesetzes über die Wirtschaftlichkeit und Qualität der Leistungen zu überwachen. Namentlich sind folgende Angaben zu machen: a. Art der Tätigkeit, Einrichtung und Ausstattung, Rechtsform; b. Anzahl und Struktur der Beschäftigten und der Ausbildungsplätze; c. Anzahl und Struktur der Patientinnen und Patienten in anonymisierter Form; d. Art, Umfang und Kosten der erbrachten Leistungen; e. Aufwand, Ertrag und finanzielles Betriebsergebnis; f. medizinische Qualitätsindikatoren

8 Schweizer S, Trümpler R, Werder G. Wild West bei Wirtschaftlichkeitsverfahren? Schweiz Ärztezeitung. 2017;98(12):382-4.

Kessler T, D'Angelo M, Trittin A. Neue Wege bei der Wirtschaftlichkeitsprüfung. Schweiz Ärztezeitung. 2017;98(7):208-9. Krankenkassen, santésuisse und Wirtschaftlichkeitsverfahren, die keine sind ... Schweiz Ärztezeitung. 2009;90(1/2)

Romanens M. Schadet das Ärzterating der medizinischen Grundversorgung? Schweiz Ärztezeitung. 2005;86(32/33):1911-4. 